\title{
Parafusos bioabsorvíveis na reparação de fraturas experimentais de sesamóides proximais em eqüinos
}

\author{
Bioabsorbable screws in the healing of experimentally induced fractures of the proximal sesamoid bone \\ in horses
}

\author{
Marcelo Damas Pyles $^{\mathrm{I}^{*}}$ Ana Liz Garcia Alves" Carlos Alberto Hussni" Armen Thomassian ${ }^{\mathrm{II}}$ \\ José Luiz de Mello Nicoletti" ${ }^{I I}$ Marcos Jun Watanabe ${ }^{\mathrm{I}}$
}

\section{RESUMO}

Avaliou-se a eficácia dos implantes bioabsorvíveis de ácido polilático na fixação interna de fraturas induzidas no osso sesamóide proximal de eqüinos. Osteossínteses foram realizadas em oito eqüinos, distribuídos em dois grupos de quatro animais conforme o implante: aqueles que receberam implantes bioabsorvíveis formaram o grupo GI e os que receberam implantes metálicos do grupo GII. O monitoramento radiográfico foi realizado no $30^{\circ}$ dia (D30), no 60ํ dia (D60), no 90o dia (D90) e no 120 dia (D120) pósoperatório. Ao exame radiográfico no D30, observou-se preenchimento do foco de fratura de aspecto radiopaco, porém com densidade reduzida nos animais do grupo GII. Tal preenchimento às análises clínica e radiográfica apresentou qualidade superior no grupo GI, quando comparado com o grupo GII. No D120, observou-se, nos animais de ambos os grupos, ao exame radiográfico, o preenchimento do foco de fratura por tecido radiopaco, indicando a reparação da fratura em sua quase totalidade. Contudo, no GI, observaram-se pontos de esclerose ao redor do implante bioabsorvível e, ao exame físico, ausência de claudicação e, nos animais do GII, diminuição da flexão metacarpo-falangeana e claudicação de grau I. Os implantes bioabsorvíveis de PLLA mostram-se eficazes na fixação interna de fraturas induzidas dos ossos sesamóides proximais de eqüinos; os animais submetidos à redução da fratura com implantes bioabsorvíveis apresentaram ao final do experimento grau de claudicação menor quando comparados com os animais que receberam implantes metálicos. À análise radiográfica, os implantes bioabsorvíveis de PLLA possibilitaram remodelamento ósseo de melhor qualidade, quando comparados com os implantes metálicos.
Palavras-chave: osteossíntese, sesamóide proximal lateral, parafusos bioabsorvíveis, equino.

\begin{abstract}
This study evaluated the efficacy of bioabsorbable polylactic acid implants in internal fixation of induced fractures of the proximal sesamoid bone in horses. Eight horses underwent osteosynthesis; four received bioabsorbable implants (group GI) and other four received metallic implants (group GII). Fractures were experimentally induced by means of osteotomy on the midportion of the lateral proximal sesamoid bone. Subsequently, the fragments were fixed with screws and the horses were $x$-rayed and a fiberglass cast was placed (D0). The cast was removed for radiographic examination on the $30^{\text {th }}$ postoperative day (D30), with radiographic follow up on the $60^{\text {th }}$ (D60), 90 $0^{\text {th }}$ (D90) and $120^{\text {th }}$ (D120) days. Radiographs taken on D30 showed radiodense filling of the fracture site; however, horses from group GII had a less dense fracture line; clinical and radiographic analysis showed a better fracture filling in group GI when compared to Group GII. On D120 radiographic examination revealed radiodense filling of the fracture site in both groups, indicating almost complete fracture healing. However, sclerotic spots were observed around the bioabsorbable implant in GI; on physical examination, horses from group GI were not lame, while horses from group GII had reduced flexion of the metacarpo-phalangeal joint and grade I lameness.
\end{abstract}

Key words: osteosynthesis, proximal sesamoid bone, bioabsorbable implants, equine.

\footnotetext{
ICurso de doutorado, Departamento de Cirurgia e Anestesiologia Veterinária, Faculdade de Medicina Veterinária e Zootecnia (FMVZ), Universidade Estadual Paulista (UNESP), Botucatu SP, Brasil. *Endereço para correspondência: Rua Sete, 29, Cond. Colinas do Paraíso, 18608-151, Botucatu, SP, Brasil. E-mail: marcelodpyles@zipmail.com.br.

"Departamento de Cirurgia e Anestesiologia Veterinária, FMVZ, UNESP, Botucatu, SP, Brasil.
} 


\section{INTRODUÇÃO}

Dentre as lesões relacionadas ao aparelho locomotor dos eqüinos atletas, as fraturas dos ossos sesamóides proximais apresentam alta incidência em cavalos submetidos ao estresse de modalidades esportivas em que se atingem altas velocidades, como ocorre em cavalos de corrida (HONNAS, 1992; STASHAK, 1994; PASQUINI et al., 1995; BERTONE, 1996; RUGGLES \& GABEL, 1998; RIEGEL \& HAKOLA, 1999; TORRE \& MOTTA, 1999).

Um dos tratamentos preconizados para as fraturas dos ossos sesamóides proximais refere-se à fixação interna dos fragmentos por meio da aplicação de implantes (parafusos). Neste sentido, os implantes metálicos fazem parte do arsenal de materiais cirúrgicos disponíveis aos cirurgiões ortopedistas há anos. Contudo, existem relatos de complicações relacionadas ao seu emprego como: a reação do organismo ao material, a interferência nas técnicas de acompanhamento pós-operatórias por imagem e a restrição do crescimento ósseo fisiológico em pacientes jovens (PIETRZAK et al., 1996). Esses implantes possuem a propriedade de terem eficácia no organismo até o período em que há a consolidação da fratura, recomendando-se sua remoção após esse período. Além disso, o efeito indesejável mais preocupante, relacionado com seu emprego, é o desenvolvimento de osteopenia cortical induzida pelo estresse do material no tecido ósseo (BÖSTMAN, 1991).

Visando à diminuição dos possíveis efeitos adversos do emprego dos implantes metálicos nas osteossínteses, recentemente, disponibilizaram-se implantes confeccionados com materiais bioabsorvíveis, que possuem como propriedades o favorecimento de intenso suprimento sanguíneo ao foco de fratura, decorrente da absorção gradativa do material pelo organismo, auxiliando assim o processo de reparação óssea (BÖSTMAN et al., 1992; STEFFANUS, 1997).

Os implantes bioabsorvíveis, comparativamente aos metálicos, proporcionam vantagens como a menor reação de tecidos moles e a ausência da necessidade de remoção dos implantes. A biodegradação do implante permite ainda transferir estresse gradual ao osso em consolidação e assim estimular o remodelamento ósseo completo após a lesão (HOVIS \& BUCHOLZ, 1997). Além disso, segundo FURUKAWA et al. (2000), os implantes bioabsorvíveis previnem a atrofia induzida pelo estresse e enfraquecimento do osso fixado, que são comumente observados na fixação rígida por parafusos metálicos.
Segundo BÖSTMAN (1991), os implantes bioabsorvíveis podem ser confeccionados de diversos materiais, sendo que os de ácido polilático (PLLA) possuem a propriedade de manter a resistência às tensões até que ocorra a consolidação da fratura. A taxa de degradação do PLLA depende do peso molecular do material, da técnica de processamento, da geometria, do tamanho do implante e do local da implantação (BÖSTMAN et al., 1995; JUKKALA-PARTIO et al., 2001). Em estudos realizados em humanos, foi observada a absorção pelo período de quatro a cinco anos, de maneira gradual e possibilitando a substituição do implante por tecido ósseo. Em eqüinos, ainda não foi determinado o período necessário para que a absorção total ocorra (FIELD, 1997; STEFFANUS, 1997).

Considerando as características dos implantes de polímeros de ácido lático tais como boa compatibilidade óssea, discreta flexibilidade que oferece após implantação, permitindo a ação de estresse gradual ao osso em reparação, o que favorece seu remodelamento após uma lesão, e a não necessidade de remoção uma vez que é absorvido, a presente investigação experimental teve como objetivos avaliar a técnica cirúrgica utilizada para a osteotomia e a osteossíntese do sesamóide proximal lateral e a eficácia dos parafusos bioabsorvíveis de PLLA na fixação interna de fraturas de tal osso.

\section{MATERIAL E MÉTODOS}

Foram utilizados oito eqüinos adultos, sem raça definida, machos e fêmeas, alimentados com feno de capim “coast-cross”, ração comercial para eqüinos e suplemento mineral. Após avaliações clínica e radiográfica, os animais foram submetidos no mesmo tempo cirúrgico à osteotomia (dia D0) e osteossíntese com parafuso, conforme o grupo experimental: grupo I (GI - quatro animais), parafuso bioabsorvível de PLLA; e grupo II (GII - quatro animais), parafuso metálico, ambos com 4,5mm de diâmetro. Após o procedimento cirúrgico, os animais de ambos os grupos foram monitorados por meio de exames físicos e radiográficos a cada 30 dias (D30, D60, D90) até o período de 120 dias (D120).

Com o animal sob anestesia geral inalatória e decúbito lateral, foi realizada a abordagem cirúrgica do osso sesamóide proximal lateral através de uma incisão semicircular de pele na face lateral da articulação metacarpo-falangeana esquerda, sobre o ligamento suspensório. O plexo neurovascular foi deslocado caudalmente e realizada incisão dos ligamentos sesamoídeos colaterais para a visibilização da 
superfície articular do osso sesamóide proximal lateral. Realizou-se a osteotomia no sentido transversal na região média do osso, utilizando-se broca, serra circular odontológica e osteótomo. Com o auxílio de um fórceps, os dois fragmentos foram justapostos visando à fixação, para em seguida criar-se um trajeto com uma broca de 3,5mm de diâmetro na direção da base ao ápice do osso sesamóide. Através desse trajeto, foi inserido o parafuso bioabsorvível $^{\mathrm{a}}$ (GI) ou o parafuso metálico (GII). A articulação foi irrigada com 2L de solução fisiológica $0,9 \% \mathrm{NaCl}$, visando à remoção dos fragmentos ósseos. O tecido subcutâneo foi reduzido com padrão de sutura contínuo tipo cerzidura, com fio vicryl violeta 2-0, e a pele com padrão de sutura simples separado e fio de náilon 2-0. Uma bandagem foi aplicada no local da incisão, colocada ferradura com talonete de $5 \mathrm{~cm}$ com a finalidade de se evitar fratura do gesso e facilitar o apoio do membro, o qual foi imobilizado com gesso sintético ${ }^{c}$ desde o casco até o terço proximal do osso metacarpiano. Após a recuperação da anestesia os animais foram medicados com fenilbutazona (2,2 $\mathrm{mg} \mathrm{kg}^{-1}$, via intramuscular) a cada 12 horas durante quatro dias e penicilina benzatina (30.000 $\mathrm{UI} \mathrm{kg}^{-1}$, via intramuscular) a cada 72 horas, totalizando três aplicações.

No D30, foram removidos a imobilização com gesso sintético e os pontos da pele, e o ferrageamento foi substituído por ferradura convencional. Até o D60 os animais foram mantidos com bandagem e em baias individuais. Após este período, foram soltos em piquetes, onde permaneceram até o D120.

Por meio do exame físico, foram avaliados sinais como aumento de temperatura na articulação, aumento de volume local, sensibilidade dolorosa à palpação da articulação e a presença de claudicação, quantificada em escores de I a IV, segundo STASHAK (1994). As radiografias foram realizadas com aparelho portátil $^{\mathrm{d}}$ para exame radiográfico nas projeções: dorsopalmar, látero-medial, dorsolateral-palmaromedial e dorsomedial-palmarolateral e classificadas de acordo com escores. A técnica radiográfica utilizada foi de 53KV e 6,0mAs, com a distância foco-filme de um metro.

Radiografias do grupo GI: quanto à reparação da linha de fratura: 1, 2, 3, ou 4, sendo intensa, moderada, discreta ou ausência de linha radioluscente, respectivamente; preenchimento ósseo no trajeto do parafuso: 1, 2, 3 ou 4, sendo intensa, moderada, discreta ou ausência de linha de fratura, respectivamente; presença de canais vasculares no osso sesamóide: 1 , 2, 3, ou 4, sendo ausência, discreta, moderada ou intensa evidência da presença de canais vasculares, respectivamente; reação osteoproliferativa na borda abaxial do osso sesamóide: 1, 2, 3 ou 4, sendo ausência, discreta, moderada ou intensa presença de reação osteoproliferativa, respectivamente. Para classificação das radiografias dos animais do grupo GII, foram utilizados os mesmos escores do grupo GI, com exceção da classificação quanto ao preenchimento ósseo no trajeto do parafuso.

Foi utilizado o teste de Friedman para comparação dos momentos em cada grupo, o teste de Wilcoxon para comparação dos grupos em cada momento (FISHER \& BELLE, 1993) e o nível de significância utilizado foi de $5 \%$.

\section{RESULTADOS E DISCUSSÃO}

A osteossíntese do sesamóide proximal empregada foi a mesma descrita por HONNAS (1992) e RIEGEL \& HAKOLA (1999) e foi eficaz na fixação dos fragmentos do osso sesamóide proximal lateral criados experimentalmente. A introdução do implante metálico foi um procedimento simples e rápido, devido a este ser um parafuso muito resistente. Já, quando utilizado o parafuso bioabsorvível, a duração da técnica cirúrgica foi mais prolongada devido à maleabilidade do implante, exigindo dessa maneira maiores cuidados na sua implantação.

Durante a manipulação dos parafusos, notou-se que um dos aspectos limitantes dos polímeros bioabsorvíveis é sua menor resistência comparada aos metais, como afirmaram PIETRZAK et al. (1996). Além disso, os parafusos bioabsorvíveis de PLLA têm um custo 30 vezes superior ao dos metálicos convencionais, o que pode vir a ser outro fator limitante. Porém, deve-se relevar o custo e o risco anestésico de uma segunda cirurgia para remoção do parafuso, quanto se opta pelo emprego do implante metálico, corroborando com FIELD (1997) e STEFFANUS (1997).

Imediatamente após a remoção do gesso no D30, observaram-se, nos animais de ambos os grupos, ferida cirúrgica com completa reparação, sensibilidade dolorosa ao apoiarem o membro abordado cirurgicamente e claudicação grau III. Ainda, com relação à claudicação, no D60, nos animais do GI, a claudicação foi reduzida a grau I intermitente, e esta no D90 foi considerada ausente; já no GII, no mesmo período, observou-se grau II. No D120, no grupo GII, um animal apresentou claudicação grau II, um animal grau I e dois animais não apresentaram claudicação. Nos dois animais claudicantes, observaram-se restrição na flexão da articulação metacarpo-falangeana e desmite do ligamento suspensório, diagnosticada por exame clínico e ultra-sonográfico. Por outro lado, no grupo GI, apenas um animal apresentou claudicação grau I e sem diminuição na flexão da articulação metacarpofalangeana e/ou desmite. 
A claudicação provavelmente estaria relacionada com lesão do ligamento suspensório, como foi descrito por HONNAS (1992). Uma complicação pós-operatória que pode ocorrer após a redução e fixação interna de fraturas é a dor no local do implante metálico, o que também explicaria o fato de os animais claudicarem. A ocorrência de desmite foi relacionada com reação abaxial no osso sesamóide estudado, a qual foi mais evidente no grupo GII.

BÖSTMAN (1991) afirmou que dos efeitos adversos dos materiais metálicos, o mais importante é a osteopenia cortical induzida pelo estresse causado pelo implante e irritação crônica dos tecidos moles adjacentes. PIETRZAK et al. (1996) relataram que estes parafusos podem também atuar como corpo estranho, causando reação inflamatória. Estas citações corroboram com o observado nos animais 02 e 03 do grupo GII do presente estudo.

Apesar de não haver diferença significante entre os escores dos grupos GI e GII com relação à linha de fratura (Figura 1A), observou-se no GI moderada linha radioluscente no D30, discreta linha radioluscente no D90 e ausência de linha radioluscente no D120 (Figuras 2A, B, C, D). Entretanto, no grupo GII, o preenchimento da linha de fratura ocorreu de maneira irregular, podendo ser visualizadas algumas pontes radiopacas ligando os dois fragmentos e, ao redor destas pontes, pontos radioluscentes (Figuras 3A, B, C, D).

Foi observado preenchimento ósseo no trajeto do parafuso aos 120 dias (Tabela 1), quando foram utilizados os parafusos bioabsorvíveis na osteossíntese. Este fato pode ter ocorrido não devido à sua absorção, mas devido à diminuição do seu peso molecular, o que leva à perda de suas características geométricas. BÖSTMAN (1991) e PELTONIEMI et al. (1999) ressaltaram que a resistência de um implante de poliéster declina antes da degradação macroscópica começar.

Em ambos os grupos estudados, observou-se a presença de canais vasculares, porém sem diferença significante entre eles (Figura 1B). Este fato provavelmente se deve à fase inflamatória que ocorre no processo de reparação óssea de qualquer fratura, independentemente do método de fixação utilizado, como descreveram PIERMATTEI \& FLO (1999). Porém, nos animais do grupo GI, sugeriu-se que o processo inflamatório ósseo ocorreu de forma discreta, prolongada e decrescente, podendo ser

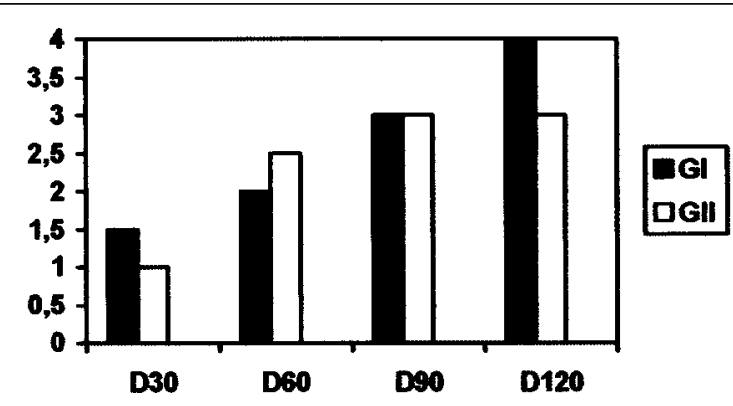

Figura 1A - Medianas dos escores de reparação da linha de fratura do osso sesamóide proximal lateral submetido à osteossíntese dos animais do grupo GI (parafuso bioabsorvível) e grupo GII (parafuso metálico) à análise radiográfica em variados dias após a cirurgia (D30, D60, D90 e D120).

D30 - 30 dia após a cirurgia; D60 - 60 dia após a cirurgia; D90 - 90 dia após a cirurgia; D120 - 120 dia após a cirurgia.

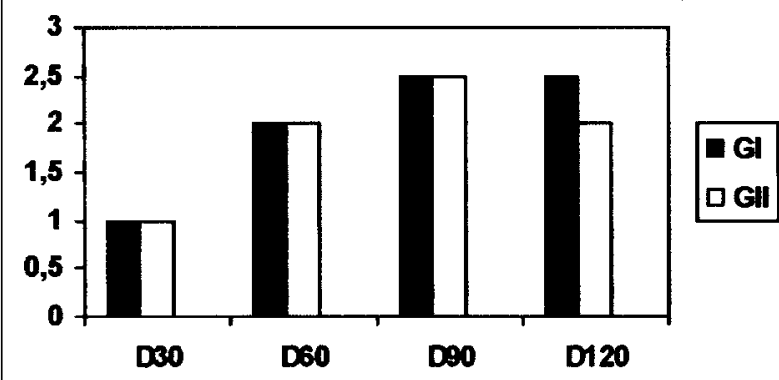

Figura 1B - Medianas dos escores de presença de canais vasculares do osso sesamóide proximal lateral submetido à osteossíntese dos animais do grupo GI (parafuso bioabsorvível) e grupo GII (parafuso metálico) à análise radiográfica em variados dias após a cirurgia (D30, D60, D90 e D120).

D30 - 30 dia após a cirurgia; D60 - 60 dia após a cirurgia; D90 - 90 dia após a cirurgia; D120 - $120^{\circ}$ dia após a cirurgia.

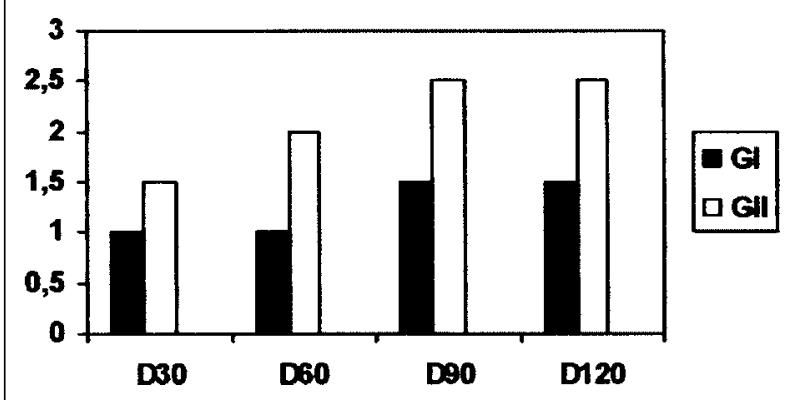

Figura 1C - Medianas dos escores de reação osteoproliferativa no bordo abaxial do osso sesamóide proximal lateral submetido à osteossíntese dos animais do grupo GI (parafuso bioabsorvível) e grupo GII (parafuso metálico) à análise radiográfica em variados dias após a cirurgia (D30, D60, D90 e D120).

D30 - 30 dia após a cirurgia; D60 - 60 dia após a cirurgia; D90 - $90^{\circ}$ dia após a cirurgia; D120 - $120^{\circ}$ dia após a cirurgia. 


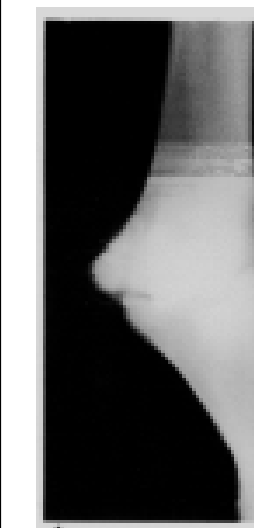

$\overline{\mathbf{A}}$

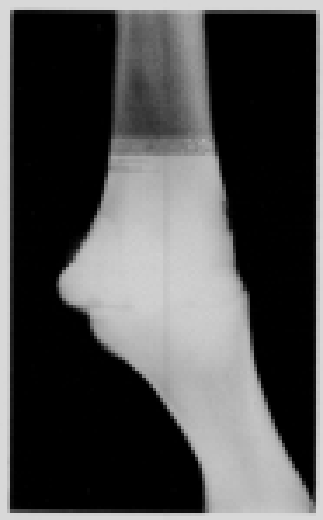

B

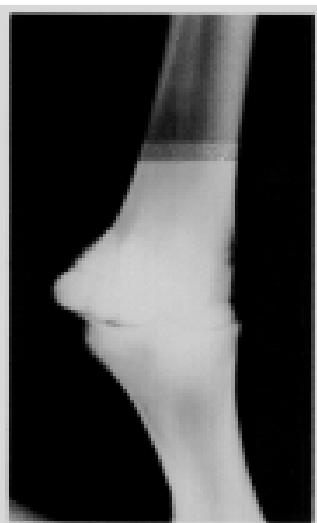

C

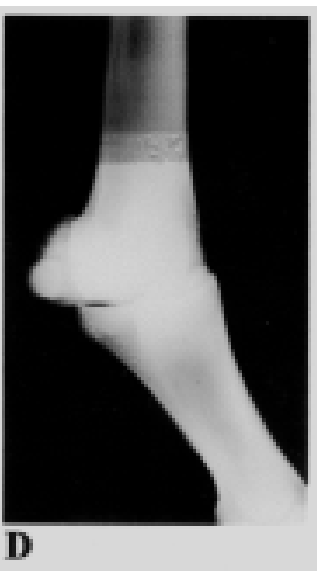

D

Figura 2 - Seqüência de imagens radiográficas (A, B, C e D) de um animal do grupo GI, no D30, D60, D90 e D120, respectivamente.

visibilizados no osso sesamóide proximal fraturado canais vasculares dilatados com maior diâmetro e em maior número entre o D30 e D60 e, em menor freqüência e diâmetro, no D90, estando presentes ainda no D120. Isto mostra uma melhor cicatrização e remodelação do osso sesamóide nas avaliações radiográficas no grupo GI do que no grupo GII. Nos animais do grupo GII, o processo inflamatório ósseo ocorreu de forma mais aguda e com menor período de duração, prejudicando as características do osso sesamóide observadas nas avaliações radiográficas, as quais evidenciaram intensa reação abaxial no osso em questão.

Na avaliação da reação osteoproliferativa da borda abaxial do osso sesamóide, observou-se reação expressiva (Figura 1C). Resultado semelhante foi obtido por VILJANEN et al. (1995), que observaram, após 144 dias, calo ósseo maior na fixação metálica do que na fixação com PLLA. De acordo com esses autores, os implantes de aço inoxidável utilizados na fixação são dez vezes menos elásticos do que os de PLLA, o que explicaria o fato da maior ocorrência de reação abaxial observada no grupo GII, devido ao maior estresse causado ao osso sesamóide provocado pelo parafuso metálico.

De acordo com BÖSTMAN (1991), entre os implantes de materiais bioabsorvíveis, o PLLA tem a menor taxa de degradação (meia vida de seis meses), enquanto as taxas dos copolímeros de polidextro e copolímeros de ácido polilevolático, ácido poliglicólico, ácido poliglicólico:ácido polilático e poliparadioxanone são mais rápidas. O peso molecular, a cristalinidade, a história térmica e a geometria do implante influenciam consideravelmente a degradação; uma superfície porosa fina despolimeriza muito mais rapidamente que um bloco denso. Contudo, a degradação não implica absorção imediata do implante, como foi observado nas avaliações radiográficas do presente estudo, estando de acordo com afirmações

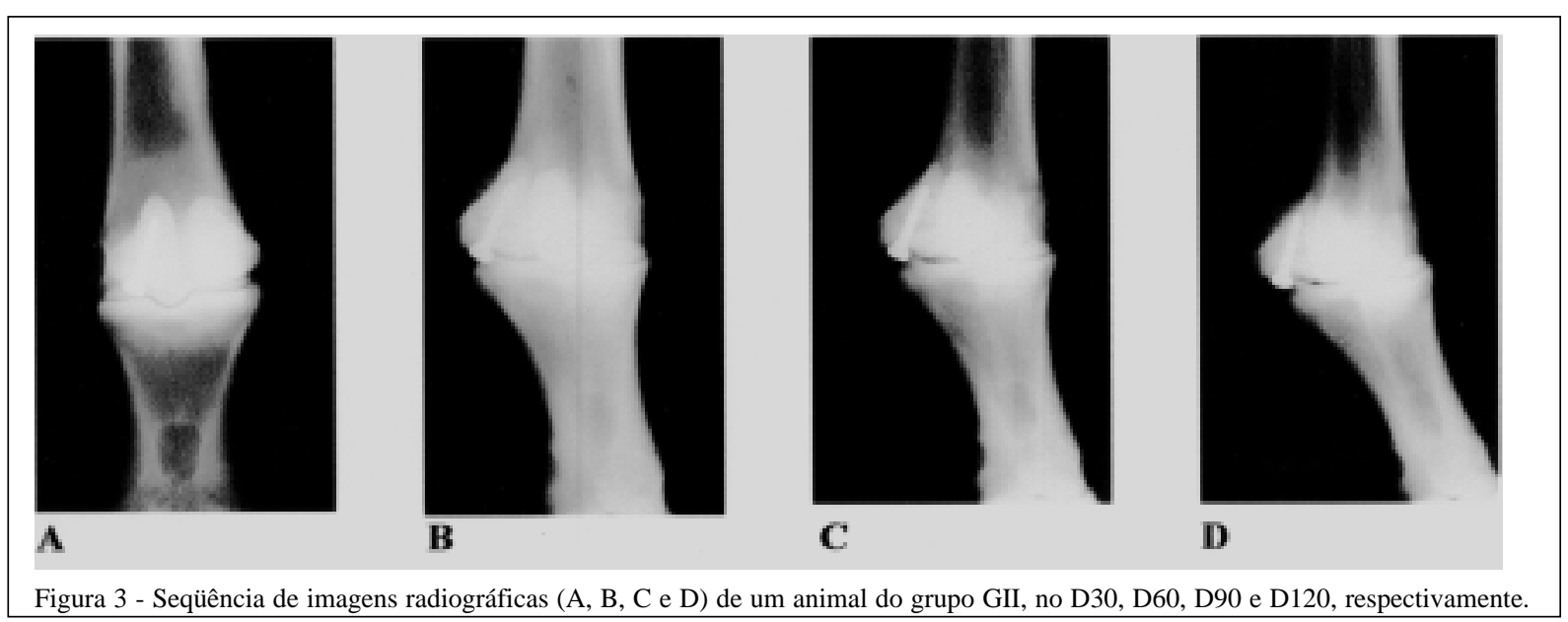

Ciência Rural, v.37, n.5, set-out, 2007. 
Tabela 1 - Mediana, $1^{\circ}$ e $3^{\circ}$ quartis referentes aos escores das radiografias dos animais do Grupo GI quanto ao preenchimento ósseo no parafuso no osso sesamóide proximal lateral submetido à osteossíntese segundo os momentos.

\begin{tabular}{cccc}
\hline Momento & Mediana & $1^{\circ}$ Quartil & $3^{\text {o }}$ Quartil \\
\hline D30 & $1,0^{\mathrm{b}}$ & 1,0 & 1,5 \\
D60 & $2,0^{\mathrm{a}}$ & 2,0 & 2,5 \\
D90 & $3,0^{\mathrm{a}}$ & 2,5 & 3,0 \\
D120 & $3,0^{\mathrm{a}}$ & 3,0 & 3,0 \\
\hline
\end{tabular}

${ }^{\mathrm{a}, \mathrm{b}}$ Momentos representados pelas medianas seguidas de mesma letra dentro de cada grupo não diferem estatisticamente $(\mathrm{P}>0,05)$. D30 - 30 dia após a cirurgia; D60 - 60 dia após a cirurgia; D90 $90^{\circ}$ dia após a cirurgia; D120 - $120^{\circ}$ dia após a cirurgia.

de BÖSTMAN (1991), BÖSTMAN et al. (2000) e BÖSTMAN \& PIHLAJAMÄKI (2000). Em nosso experimento, no D120 de todos os animais do grupo GI, ainda observou-se presença do parafuso bioabsorvível, do que pode se interpretar que, até os 120 dias de pós-operatório, o parafuso não havia sido absorvido, corroborando com PIETRZAK et al. (1996) que afirmavam que muitos dos materiais bioabsorvíveis são eliminados do organismo em aproximadamente um ano a um ano e meio. Porém, o PLLA pode requerer vários anos para a completa absorção, devido a estrutura do polímero possuir alto peso molecular e cristalinidade.

De acordo com BERGSMA et al. (1996), BÖSTMAN \& PIHLAJAMÄKI (2000) e MIDDLETON \& TIPTON (2000), o PLLA pode requerer mais que cinco anos para ser completamente bioabsorvido. YUEHUEI et al. (1998) também afirmaram que o PLLA é altamente cristalino e reabsorvido lentamente. Este é reabsorvido dentro de vários anos, restando cristais que podem provocar uma resposta inflamatória a corpo estranho. JUKKALA-PARTIO et al. (2001) citam que existem certas limitações nos estudos que necessitem de longo período de avaliação. Na opinião dos autores do presente experimento, um estudo clínico com PLLA deveria ser analisado por pelo menos cinco anos depois de realizado o implante para que conclusões referentes à absorção pudessem ser tiradas com mais exatidão. Porém, no período de avaliação do presente estudo foi possível obter resultados satisfatórios na fixação de fraturas de ossos sesamóides proximais de eqüinos com implantes de PLLA.

\section{CONCLUSÕES}

Nas condições experimentais utilizadas, pode-se concluir que a técnica cirúrgica utilizada para a osteotomia e a osteossíntese experimental de sesamóides proximais é exeqüível, possibilitando acesso fácil ao osso sem apresentar complicações. Além disso, conclui-se que os implantes bioabsorvíveis de PLLA são eficazes na fixação interna de fraturas induzidas dos ossos sesamóides proximais de eqüinos, sendo que animais submetidos à redução da fratura com implantes bioabsorvíveis apresentam, ao final do experimento, grau de claudicação menor, quando comparados com os que recebem implantes metálicos. Por fim, pela análise radiográfica, implantes bioabsorvíveis de PLLA possibilitam remodelamento ósseo de melhor qualidade quando comparados com implantes metálicos.

\section{AGRADECIMENTOS}

À Fundação de Amparo à Pesquisa do Estado de São Paulo - FAPESP, pelo auxílio financeiro. Processo número 00/12544-5.

\section{FONTES DE AQUISIÇÃO}

a.SmartScrew - Bionx Implants Ltd, Finlandia.

b.ASIF - Synthes, São Paulo.

c.Scothcast - 3M, EUA.

d.Jockey 90 - FNX, Rio de Janeiro.

\section{COMITÊ DE ÉTICA E BIOSSEGURANÇA}

O presente trabalho foi aprovado pela Câmara de Ética em Experimentação Animal de acordo com os Princípios Éticos na Experimentação Animal (COBEA), protocolo número 004/2002-CEEA.

\section{REFERÊNCIAS}

BERGSMA J.E. et al. Biocompatibility of intraosseously implanted predegraded poly (lactide): an animal study. Journal of Material Science: Materials in Medicine, Dordrecht, v.7, n.1, p.1-7, 1996.

BERTONE A.L. Fractures of the proximal sesamoid bones. In: NIXON, A.J. Equine fracture repair. Philadelphia: Saunders, 1996. p.163-171.

BÖSTMAN O. Absorbable implants for the fixation of fractures. Journal of Bone and Joint Surgery, Boston, v.73, n.1, p.148-153, 1991.

BÖSTMAN O. et al. Degradation and tissue replacement of an absorbable polyglycolide screw in the fixation of rabbit femoral osteotomies. Journal of Bone and Joint Surgery, Boston, v.74, n.7, p.1021-1031, 1992.

BÖSTMAN, O. et al. Clinical biocompatibility and degradation of polylevolactide screws in the ankle. Clinical Orthopaedics and Related Research, Philadelphia. n.320, p.101-109, 1995.

BÖSTMAN O.; PIHLAJAMÄKI H. Clinical biocompatibility of biodegradable orthopaedic implants for internal fixation: a review. Biomaterials, Surrey, v.21, n.24, p.2615-2621, 2000. 
BÖSTMAN O. et al. Response of articular cartilage and subchondral bone to internal fixation devices made of poly-Llactide: a histomorphometric and microradiophic study on rabbits. Biomaterials, Surrey, v.21, n.24, p.2553-2560, 2000.

FIELD J.R. Bioabsorbable screws. The Horse, Lexington, v.14, p.45-49, 1997.

FISHER, L.D., BELLE, G.V. Biostatistics a methodology for the health sciences. New York: Wiley-interscience, 1993. 991p.

FURUKAWA T. et al. Biodegradation behavior of ultra-highstrength hydroxyapatite/poly (L-lactide) composite rods for internal fixation of bone fractures. Biomaterials, Surrey, v.21, n.9, p.889-898, 2000.

HONNAS C.M. Surgical treatment of selected musculoskeletal disorders of the forelimb. In: AUER J.A. Equine surgery. Philadelphia: Saunders, 1992. p.985-1054.

HOVIS W.D.; BUCHOLZ R.W. Polyglycolide bioabsorbable screws in the treatment of ankle fractures. Foot \& Ankle International, Baltimore, v.18, n.3, p.128-131, 1997.

JUKKALA-PARTIO K. et al. Biodegradation and strength retention of poly-L-lactide screws in vivo. An experimental long-term study in sheep. Annales Chirurgiae et Gynaecologiae, Helsingfors, v.90, n.3, p.219-224, 2001.

MIDDLETON J.C.; TIPTON A.J. Synthetic biodegradable polymers as orthopedic devices. Biomaterials, Surrey, v.21, n.23, p.2335-2346, 2000.

PASQUINI C. et al. Guide to equine clinics lameness. 3.Print. Texas: Sudz Publishing, 1995. V.2.

PELTONIEMI H.H. et al. SR-PLLA and SR-PGA miniscrews: biodegradation and tissue reactions in the calvarium and dura mater. Journal of Cranio-Maxillofacial Surgery, Kidlinton, v.27, n.1, p.42-50, 1999.

PIERMATTEI D.L.; FLO G.L. Fraturas: classificação, diagnóstico e tratamento. In: ____. Ortopedia e tratamento das fraturas dos pequenos animais. 3.ed. São Paulo: Manole, 1999. p.24-138.

PIETRZAK W.S. et al. Bioresorbable implants - practical considerations. Bone, New York. v.19, suppl. 1, p.109S-119S, 1996.

RIEGEL R.J.; HAKOLA S.E. Illustraded atlas of clinical equine anatomy and common disorders of the horse. 3.Print. Ohio: Equistar, 1999. 260p.

RUGGLES A.J.; GABEL A.A. Injuries of the proximal sesamoid bones. In: WHITE N.A.; MOORE J.N. Current techniques in equine surgery and lameness. 2.ed. Philadelphia: Saunders, 1998. p.403-408.

STASHAK T.S. Claudicação em eqüinos segundo Adams. 4.ed. São Paulo: Roca, 1994. 943p.

STEFFANUS, D. Bone screws advance fracture repair. Equine Athlete, Santa Barbara, v.10, p.12, 1997.

TORRE F.; MOTTA M. Incidence and distribution of 369 proximal sesamoid bone fractures in 354 Standardbred horses (1984-1995). Equine Practice, Santa Barbara, v.21, n.8, p.612, 1999.

VILJANEN J. et al. Bone changes after experimental osteotomies fixed with absorbable self-reinforced poly-L-lactide screws or metallic screws studied by plain radiographs, quantitative computed tomography and magnetic resonance imaging. Biomaterials, Surrey, v.16, n.17, p.1353-1358, 1995.

YUEHUEI H. et al. Fixation of osteotomies using bioabsorbable screws in the canine femur. Clinical Orthopaedics \& Related Research, Philadelphia, v.355, p.300-311, 1998. 